\title{
Integración de la educación interprofesional: autodiagnóstico para programas de salud
}

\author{
Integration of Interprofessional Education: Self-Assessment for health care programs
}

\author{
Silvia Lizett Olivares Olivares', Claudia Susana López Cruz²
}

\section{Resumen}

Introducción: la educación interprofesional se produce cuando dos o más profesionales se conocen y se relacionan entre sí para permitir una colaboración eficaz con la intención de mejorar los resultados de salud. Diferentes marcos de referencia de identidad profesional y competencias han establecido la importancia de este tipo de formación. Sin embargo, cada programa puede contar con diferentes niveles de implementación para integrarlo en el mismo. Método: el objetivo del presente trabajo fue desarrollar un instrumento de autodiagnóstico sobre Integración de la Educación Interprofesional en los programas de salud, considerando su formalización y despliegue. Se utilizó una validación de expertos para el diseño del instrumento y una prueba piloto con directivos de cinco programas de salud. Resultados: el instrumento consta de 12 ítems para valorar si los alumnos cuentan con condiciones formativas interprofesionales: 1) Identifican, 2) Colaboran, 3) Toman decisiones y 4) Construyen soluciones. Para ello la institución educativa puede A. Definir, B. Comunicar, C. Formar, D. Evaluar o E. Mejorar las intervenciones formativas para garantizar una implementación adecuada de dichos propósitos. El modelo resultante es una guía taxonómica que permite identificar la formalización de la implementación cada una de las competencias en un programa de salud. Conclusiones: el instrumento propuesto cuenta con contenidos comprensibles y adecuados para cumplir con sus propósitos de autodiagnóstico en la formalización en la implementación de competencias interprofesionales. Sin embargo, más que un carácter estrictamente incremental, confiere una visión dinámica en la cual pueden implementarse escalas superiores sin haber cerrado un ciclo de mejora completo en las competencias previas.

Palabras clave: interprofesional; identidad; autodiagnóstico; competencias, integración.

\begin{abstract}
Introduction: Interprofessional education occurs when two or more professions learn about, from, and with each other to enable effective collaboration and improve health outcomes. Different frameworks of professional identity and competencies have established the importance of interprofessional education. However, each program may have different levels of formalisation or implementation. Methods: The objective of this work was to develop a self-assessment instrument regarding the interprofessional education integration for health care programs. An expert validation was applied for the instrument design, and the heads of five health programs conducted a pilot test. Results: The instrument consists of 12 items to assess interprofessional education: 1) Identify, 2) Collaborate, 3) Make decisions, and 4) Build solutions. The educational institution can A. Define, B. Communicate, C. Train, D. Evaluate, or E. Improve educational interventions to ensure adequate implementation. The resulting model is a taxonomic guide that allows identifying the formalisation of the implementation of each of the competencies in a health care undergraduate program. Conclusions: The proposed self-assessment has understandable and adequate content to address its purposes. However, more than a strictly incremental approach, it confers a dynamic vision in which higher scales can be implemented without closing a complete improvement cycle in the previous competencies.
\end{abstract}

Keywords: interprofessional, identity, self-assessment, competence, integration.

Fecha de envío: 2021-09-01- Fecha de aceptación: 2021-12-22

\section{Introducción}

La educación interprofesional se produce cuando dos o más profesiones se conocen y se relacionan entre sí para permitir una colaboración eficaz con la intención de mejorar los resultados de salud (WHO, 2010). Para que en la práctica se pueda centrar la atención en el paciente, es indispensable incorporar en los programas de salud

(1) Tecnológico de Monterrey, Escuela de Medicina y Ciencias de la Salud

(2) Tecnológico de Monterrey, Escuela de Humanidades y Educación

Autor de correspondencia: solivares@tec.mx 
las competencias interprofesionales necesarias para la práctica clínica de forma que se aprecien y valoren los roles, las voces y las aportaciones de cada uno de los involucrados en el proceso.

\section{La educación interprofesional y la identidad profesional}

Si bien la formación profesional se orienta al desarrollo de conocimientos, actitudes y valores, es innegable la participación de los profesionales de salud en más de un rol al momento de su egreso, por lo cual su participación como miembro de un equipo forma parte de la identidad profesional (Cruess et al., 2019). Al respecto Fitzgerald (2020) genera un análisis del concepto de identidad profesional en las profesiones relacionadas con la salud y encuentra que se relaciona con aspectos de conocimiento disciplinar sobre sus funciones, valores éticos, identidad social, así como el reconocimiento social del gremio. Por su parte, Olivares et al. (2020) la definen como la evolución dinámica a través de la cual el alumno se reconoce a símismo como persona que forma parte de grupos sociales y académicos para ejercer su rol dentro de contextos profesionales y de esta forma generar un cambio consciente. Los autores incluyen cuatro etapas para el desarrollo de la identidad del profesionista: 1) autoconocimiento, 2) relaciones de afinidad, 3) conceptualización profesional y 4) cambio consciente las cuales forman parte de un modelo dinámico que permite la evolución de la persona para reforzar sus intereses, valores. La tabla 1 asocia los conceptos de la definición de WHO (2010) con aspectos de la identidad profesional.

\section{Competencias para la educación interprofesional}

Educar a profesionales de salud como un equipo es una necesidad imperativa para promover aspectos como diálogo, comunicación y colaboración para el beneficio del paciente. Algunas organizaciones han establecido competencias interprofesionales. Por ejemplo, el marco de referencia de CUILU (Interprofessional Learning Framework) promueve la colaboración con el usuario/cliente como eje central del servicio o atención para la orientación de logros considerando cuatro dominios: conocimiento en la práctica, práctica ética, trabajo interprofesional y reflexión del logro. Por su parte el IPEC (2016) (Interprofessional Collaborative Practice) establece competencias con las que deben de contar los profesionales de la salud. Su propuesta presenta principios deseados para la atención centrada en el paciente, la comunidad y la población, relaciones, procesos y actividades de aprendizaje apropiadas para el desarrollo del alumno para formarlo como un profesionista sensible al contexto de la práctica interprofesional.

Otros autores han valorado la inclusión de la educación o atención interprofesional a través de indicadores estándares o de calidad (Ohland et al., 2012; Zorek \& Raehl, 2013; Bogossian \& Craven, 2020), establecen parámetros para medir la calidad de la colaboración de cada miembro del equipo e introducen el CATME (Comprehensive Assessment of Team Member Effectiveness). Otros estudios incluyen la revisión de los estándares de regulación, afiliación o acreditación para determinar la importancia que le dan a la educación interprofesional. Por ejemplo, Bogossian y Craven (2020) comparan las acreditaciones de diferentes profesiones de salud en Australia y determinan la necesidad de impulsar su inclusión en dichos marcos de referencia. Por su parte Zorek y Raehl encuentran que hay requisitos de proceso y de competencias para las acreditaciones en Estados Unidos.

\section{Escala taxonómica de la educación interprofesional en salud}

En el diseño curricular e instruccional se utilizan comúnmente taxonomías cognitivas para establecer el nivel de complejidad del procesamiento. Es así como las taxonomías de Bloom et al. (1972) o de Marzano (2001) han sido de utilidad para establecer objetivos o intenciones educativas en programas, asignaturas o cursos. Sin embargo, estas taxonomías están limitadas a actividades de carácter individual y cognitivas (recuerdo, comprensión, análisis, utilización, metacognición). En este sentido la educación interprofesional requiere una propuesta taxonómica que permita actividades de tipo interpersonal con todos los involucrados en la prevención, cuidado y atención de un paciente. La tabla 2 propone competencias para educación interprofesional en salud a partir de la comparación con diferentes modelos de referencia, los cuales se detallan posteriormente.

\section{Identifican los roles, compromisos y responsabilidades de cada miembro del equipo interprofesional}

Conocer lo que hace otro profesional de salud, tiene su relevancia en los programas educativos que se dedican a su cuidado para la formación de la identidad profesional. Fitzgerald (2020) menciona que el desarrollo de la identidad incluye distinguir las funciones propias de las de otros profesionales, es decir, se necesita saber lo que son y lo que no. Si lo alumnos son formados de forma uniprofesional la claridad de su alcance en contextos clínicos queda limitada.

El autoconocimiento implica que el individuo tenga claridad sobre sus valores y preferencias y tome decisiones alineadas a los mismos (Olivares et al., 2020). Para lograrlo, en el Tecnológico de Monterrey se ha incluido una etapa de exploración, a través de la cual pueden experimentar las opciones profesionales dentro de la entrada a un área particular dando oportunidad al estudiante de confirmar su vocación al introducirse en sus contenidos (Olivares Olivares et al., 2021). En el caso de la entrada a salud, los alumnos tienen retos en los cuales participan profesores y alumnos de diversas disciplinas 
(medicina, nutrición, odontología, biociencias y psicología) para la prevención y detección de riesgos oportunamente. Esta etapa permite reafirmar vocaciones, así como reconocer y valorar los roles de cada una de las profesiones involucradas.

La IPEC(2016) establece competencias con las que deben de contar los profesionales de la salud. En cuanto a Conocer la competencia 2 establece que el profesional captura la conciencia de regulaciones profesionales de otras profesiones en el equipo interprofesional y las estructuras, funciones y procesos del equipo en contexto. Por su parte Gordon y Walsh (2005) incluyen como una competencia el conocimiento en la práctica, es decir el profesional debe ser consciente de las regulaciones de otras profesiones de los miembros del equipo interprofesional para lograr una atención informada. Esto es relevante para evitar prácticas discriminatorias y prejuicios sobre cada integrante del equipo de salud.

\section{Colaboran con profesionalismo con cada uno de los miembros del equipo interprofesional}

Las relaciones con múltiples miembros del equipo de salud es un elemento indispensable para el desarrollo de competencias interprofesionales tales como comunicación, negociación y manejo de conflictos. La etapa las relaciones de afinidad en el modelo dinámico de identidad profesional se caracteriza por la construcción o ampliación de redes académicas, sociales y personales que sean afines a sus intereses tanto de carácter formal como informal (Olivares et al., 2020). Según Fitzgerald (2020) y Cruess et al. (2019) el proceso de socialización es un elemento indispensable para la formación de la identidad porque se desarrollan códigos de lenguaje, valores y actitudes en relación a un grupo particular para favorecer el sentido de pertenencia. Si los alumnos se educan de forma aislada en facultades ajenas e independientes, los grupos se forman con culturas diferenciadas, las cuales, al unirlas en el mismo contexto, generan barreras y disonancias entre las mismas.

El IPEC (2016) incluye como competencia la necesidad de trabajar con otras profesiones para mantener un clima de respeto mutuo. El marco de referencia CUILU se centra en la promoción de participación de cada uno de los miembros del equipo de atención (incluyendo el paciente) en la toma de decisiones, considerando parte ética de la práctica (Gordon \& Walsh, 2005). Esto implica la valoración de voces democratizadas y abiertas. Ohland et al. (2012) miden las aportaciones de los miembros del equipo a partir del cumplimiento de sus responsabilidades en tiempo y forma a pesar de las dificultades presentadas y de la comunicación e interacción efectiva con compañeros. Según Freeland y Charania (2021), las relaciones constituyen un capital relacional que se vuelve indispensable para temas de socialización, bienestar y empleabilidad.
Dependiendo del entorno clínico de formación, este puede ser de total segregación a total integración. Según Amelung et al. (2021) un entorno vinculado o colaborativo implica la comunicación entre profesionales para referir pacientes al especialista o profesional adecuado en una atención en serie.

\section{Toman decisiones de forma conjunta para la atención centrada en el paciente en el equipo interprofesional}

La identidad profesional requiere un proceso de vivencia en entornos de alta autenticidad. La conceptualización profesional es cuando el individuo puede tomar decisiones asociadas con la profesión de su elección dentro de un contexto académico u organizacional (Olivares et al., 2020). Fitzgerald (2020) considera que la identidad se forma al pertenecer a una comunidad de práctica.

Algunas de las barreras para la colaboración eficaz entre miembros de diferentes profesiones de salud se asocian con estructuras organizacionales que determinan niveles de autoridad o responsabilidad diferenciados (Thistlethwaite et al., 2016). Cuando las facultades están segregadas en estructuras con diferentes organigramas, edificios y diseños curriculares, es difícil esperar interacción entre docentes o estudiantes. Según Amelung et al. (2021) una atención coordinada, los servicios clínicos comparten información clínica y administran la transición de pacientes entre diferentes unidades o grupos.

La IPEC (2016) incluye como competencia la comunicación con pacientes, familias, comunidades y miembros del equipo de salud de forma responsable como equipo para promover y mantener la salud en la prevención o el tratamiento. Este trabajo interprofesional, según Gordon y Walsh (2005) se centra en la valoración de las necesidades del paciente a partir de colaboración, participación y comunicación en equipos exitosos, el cual puede medirse según el cumplimiento de estándares de calidad (Ohland et al., 2012).

\section{Construyen soluciones de innovación y mejora en equipo interprofesional}

La formación profesional de líderes implica mentalidades que busquen el impacto de sus acciones. El cambio consciente como etapa dinámica de la identidad profesional, es cuando el individuo puede tomar decisiones asociadas con la profesión de su elección dentro de un contexto académico u organizacional para generar transformaciones (Olivares et al., 2020). Fitzgerald (2020) considera que los grupos consolidados tienen un reconocimiento que va más allá de los miembros del equipo, si no que trasciende a comunidades o el estatus de la sociedad.

La IPEC (2016) considera como competencia interpersonal aplicar los principios de la dinámica del equipo para desempeñarse 
efectivamente en los roles del equipo como planear, entregar y evaluar la atención centrada en el paciente y la salud poblacional considerado políticas seguras, oportunas, eficientes, efectivas y equitativas. El marco de referencia CUILU incluye la reflexión como componente para promover acciones de reciprocidad entre las profesiones, el uso de la mejor evidencia científica y la integración de mejora continua (Gordon \& Walsh, 2005). Ohland et al. (2012) miden la alineación del equipo cuando el progreso cumple con la planeación de acciones

En general, existen resultados contradictorios sobre los beneficios de la educación interprofesional. Algunos autores indican que no hay evidencia concluyente que muestren mejora en indicadores de resultados en salud individual o social (Illingworth \& Chelvanayagam, 2017). Sin embargo, Reeves et al. (2013) encuentran en 7 de 15 estudios que hubo beneficios en cuento a resultados en pacientes diabéticos, mejora en la comunicación con el paciente, mejora en el trabajo colaborativo, reducción de errores y mejora de la salud mental de los miembros del equipo de salud. Si los alumnos no son formados con un equipo interprofesional en un entorno clínico centrado en el paciente con total integración, es poco probable que se alcancen los resultados esperados. Según Amelung et al. (2021) este tipo de contextos implican servicios orientados a grupos específicos con una atención longitudinal en la cual cada uno de los miembros, incluyendo el paciente y sus familias, contribuyen en la toma de decisiones alrededor del proceso de intervención.

\section{Materiales y método}

El objetivo del presente trabajo fue desarrollar un instrumento de autodiagnóstico sobre Integración de la Educación Interprofesional en los programas de salud, considerando su formalización y despliegue. El instrumento cuenta con dos escalas: la taxonómica de competencias y los niveles de implementación. Sobre esto último, las competencias propuestas pueden tener diferentes formas de incorporarse en los planes de estudio. Harden (2007) considera 9 dimensiones: 1) incorporación en los planes de estudio, 2) comunicación a docentes y colaboradores, 3 ) estrategias educativas, 4) oportunidades de aprendizaje, 5) contenidos del curso, 6) progreso en los estudiantes, 7) evaluación, 8) ambiente de aprendizaje y 9) selección de estudiantes. Estas dimensiones son consistentes con los primeros cuatro niveles del Modelo Incremental de Calidad en donde se incluye como escalones: 1) Inicio, 2) Desarrollo, 3) Estandarización y 4) Innovación (Olivares-Olivares et al., 2016). En el inicio los programas identifican las prioridades, los procesos y la dinámica de trabajo, en el desarrollo los indicadores y estrategias de implementación. En la estandarización se administra la capacitación y la evaluación con propósitos de mejora continua. En el de innovación se promueva hacer cambios sustanciales, comparación referencial y planeación para el largo plazo.

Para propósitos del presente estudio se adoptan con modificaciones:

1) y 5) Están definidas y mapeadas en el programa. Forman parte de las intenciones y contenidos del curso.

2) Existen mecanismos de comunicación efectivos y eficientes hacia toda la comunidad académica

3) Se asegura la formación/educación en los alumnos,

6 y 7) Se evalúan sistemáticamente. Hay indicadores de progreso por alumno y programa.

Y se agrega la dimensión: Se establecen estrategias de mejora e innovación a intervalos adecuados.

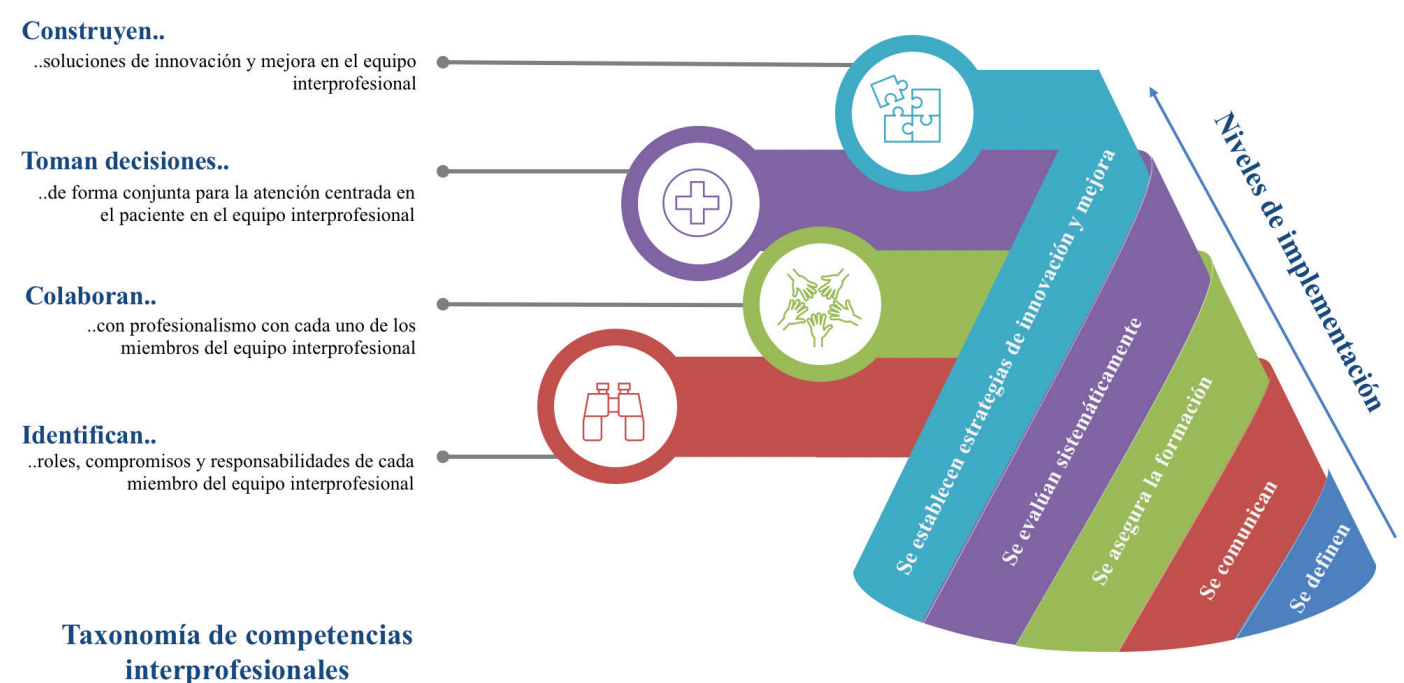

La figura 1: muestra la propuesta de esta escala en donde se va incrementando la integración del equipo en cada nivel superior de la taxonomía. 
Tabla 1: La educación interprofesional en relación a la formación de la identidad profesional.

\begin{tabular}{l|l|l}
\hline WHO (2010) & Fitzgerald (2020) & Olivares et al. (2020) \\
\hline Se conocen & Conocimientos, habilidades, acciones y comportamientos & Autoconocimiento \\
\hline Se relacionan con profesionalismo & Valores y ética & Relaciones afines \\
\hline Colaboran en el entorno clínico & Contexto y socialización & Conceptualización profesional \\
\hline Impactan al sistema de salud & Pertenencia a un grupo con reconocimiento social & Cambio consciente \\
\hline
\end{tabular}

Tabla 2: Competencias para la educación interprofesional en salud.

\begin{tabular}{|c|c|c|c|c|}
\hline $\begin{array}{l}\text { Taxonomía de competencias } \\
\text { interprofesionales }\end{array}$ & IPEC (2016) & Ohland et al. (2012) & Gordon y Walsh (2005) & Zorek y Raehl (2013) \\
\hline $\begin{array}{l}\text { Identifican los roles, com- } \\
\text { promisos y responsabilidades } \\
\text { de cada miembro del equipo } \\
\text { interprofesional }\end{array}$ & Roles y responsabilidades & $\begin{array}{l}\text { Conocimientos y } \\
\text { habilidades }\end{array}$ & $\begin{array}{l}\text { Conocimiento en la } \\
\text { práctica }\end{array}$ & $\begin{array}{l}\text { Roles y responsabilida- } \\
\text { des de los miembros de } \\
\text { equipo }\end{array}$ \\
\hline $\begin{array}{l}\text { Colaboran con profesionalismo } \\
\text { con cada uno de los miembros } \\
\text { del equipo interprofesional }\end{array}$ & $\begin{array}{l}\text { Valores y ética de la práctica } \\
\text { interprofeisonal. }\end{array}$ & $\begin{array}{l}\text { Contribución al equipo } \\
\text { Interacción con los } \\
\text { compañeros }\end{array}$ & Práctica ética & $\begin{array}{l}\text { Comunicación inter- } \\
\text { profesional/ manejo de } \\
\text { conflictos }\end{array}$ \\
\hline $\begin{array}{l}\text { Toman decisiones de for- } \\
\text { ma conjunta para la atención } \\
\text { centrada en el paciente en el } \\
\text { equipo interprofesional }\end{array}$ & $\begin{array}{l}\text { Comunicación interprofesional } \\
\text { con pacientes, familiares y } \\
\text { comunidad }\end{array}$ & Calidad y estándares & Trabajo interprofesional & $\begin{array}{l}\text { Coordinación y trabajo } \\
\text { en equipo }\end{array}$ \\
\hline $\begin{array}{l}\text { Construyen soluciones de } \\
\text { innovación y mejora en equipo } \\
\text { interprofesional }\end{array}$ & Desempeño efectivo en grupos & Alineación del equipo & Reflexión del logro & Liderazgo \\
\hline
\end{tabular}

Para que un instrumento sea considerado como validado, se deben considerar tres características principales: consistencia interna, validez y representatividad del constructo (Díaz-Barriga \& Hernández Rojas, 2002). La validez del contenido de un instrumento es el nivel de reflejo de un dominio de contenido específico que es el que se pretende medir (Hernández-Sampieri et al., 2018). Con este propósito se empleó una metodología de entrevista con expertos. Diversos autores establecen la validez de contenido de una prueba cuando el experto establece los constructos adecuados según los propósitos de esta (Downing, 2003; Martínez-Corona et al., 2020; Rodríguez Medina et al., 2021). En este sentido, formó un grupo con 5 expertos en diseño de programas con base en competencias disciplinares y transversales, así como en evaluación de las mismas. Para la selección de los expertos se emplearon diversos criterios, entre se encuentra un biorama según lo que establecen Martínez-Corona et al. (2021), como se muestra en la tabla 3.

Tabla 3: Biorama de perfil de expertos para diseño.

\begin{tabular}{l|l}
\hline Indicador & Descriptor \\
\hline Grado Académico & $80 \%$ Doctorado, 20\% maestría \\
\hline Áreas de experiencia profesional & Economía, medicina, educación, comunicación y nuevas tecnologías \\
\hline Años de experiencia en docencia e investigación (promedio) & 28,5 \\
\hline Experiencia en diseño y validación de instrumentos de investigación & 19,2 \\
\hline Artículos publicados en el área (promedio) & $>25$ artículos en promedio \\
\hline
\end{tabular}


En la primera validación con expertos, se hicieron observaciones sobre el instrumento que se fueron incorporando conforme se hacían las entrevistas, resultando en 5 iteraciones totales del mismo. En la primera iteración se hizo una descripción más detallada de la escala de valoración de cada uno de los ítems, de forma que se pudiera llegar a una escala impar coincidiendo con la escala Likert. En la siguiente iteración se recomendó desglosar mucho más cada una de las competencias de forma que se describió con mayor precisión cada una de las competencias en los elementos que las conforman.

Una vez construido el instrumento se contactó a cada uno de los expertos para validar el contenido a partir de una entrevista de forma independiente (Haynes et al., 1995; Juárez-Hernández \& Tobón, 2018). Las recomendaciones se incorporaron de forma iterativa conforme se obtuvieron las diferentes opiniones hasta llegar a la versión final del mismo. En esta versión final se incluyen 12 subdimensiones en total, con una variación en la disgregación entre 2 y 4 subdimensiones; dependiendo de la complejidad del concepto que se está pretendiendo autoevaluar. El instrumento considera la evaluación por medio de una escala tipo Likert del 1 al 5.

En la tercera iteración de los expertos en diseño curricular se recomendó considerar el perfil de los sujetos de aplicación para incluir a expertos en los temas tanto de salud como de diseño curricular. En este sentido, se administró una prueba piloto del instrumento a directores nacionales de los programas de la escuela de medicina y ciencias de la salud, de una institución de educación superior privada empleando para ello la técnica de biorama. Cada uno otorgó su consentimiento para utilizar la información obtenida para propósitos de investigación educativa (tabla 4).

Tabla 4: Biorama de perfil de expertos para validación.

\begin{tabular}{l|l}
\hline Indicador & Descriptor \\
\hline Grado Académico & $80 \%$ maestría, 20\% doctorado \\
\hline Áreas de experiencia profesional & $100 \%$ directores de programa nacionales \\
\hline Áreas disciplinares & $\begin{array}{l}\text { Psicología Clínica y de la Salud } \\
\text { Nutrición }\end{array}$ \\
& Odontología \\
Medicina & Biociencias \\
\hline Años de experiencia en áreas disciplinares & 19,4 \\
\hline
\end{tabular}

Tabla 5: Resultados de la validación con directivos de programas de salud.

\begin{tabular}{l|c}
\hline Taxonomía de competencias interprofesionales & Media \\
\hline Identifican los roles, compromisos y responsabilidades de cada miembro del equipo interprofesional & 2,25 \\
\hline Colaboran con profesionalismo con cada uno de los miembros del equipo interprofesional & 2,67 \\
\hline Toman decisiones de forma conjunta para la atención centrada en el paciente en el equipo interprofesional & 2,70 \\
\hline Construyen soluciones de innovación y mejora en equipo interprofesional & 2,73 \\
\hline
\end{tabular}

El piloto consistió en verificar si los ítems, las escalas y los constructos de las competencias quedan claros y el instrumento tiene representatividad de conceptos entre el público al que va dirigido.

\section{Resultados}

La media obtenida para cada competencia se muestra en la tabla 5 en donde se aprecia que, los valores asignados corresponden con una escala que fue comprendida y asignada respondiendo al conocimiento que tenía cada sujeto sobre el tema. Se entrevistaron a los expertos que pilotearon el instrumento posterior a la aplicación del mismo y sugirieron algunos pequeños ajustes en instrucciones, términos y condiciones, los cuales ya han sido incorporados en la versión presentada en el presente documento.
La desviación estándar por ítem tuvo un rango de 0,707 a 1,22, lo cual indica poca dispersión entre las respuestas de los expertos.

En la primera taxonomía sobre Identifican los roles, compromisos y responsabilidades de cada miembro del equipo interprofesional se obtuvo una media inferior a las otras cuatro taxonomías. El primer item "Conocen lo que hacen los otros profesionales de la salud" tuvo un promedio de 1.8, lo cual impactó en dicho resultado. En contraste, la taxonomía Construyen soluciones de innovación y mejora en equipo interprofesional fue la de mayor puntuación favorecido por el ítem “Los servicios que se proveen consideran a cada uno de los miembros, incluyendo el paciente y sus familias, para la toma de decisiones" con un promedio de 4. Al regresar con los expertos ellos indicaron que, aunque los alumnos traba 
jan juntos en la atención de pacientes, no se dedica tiempo en los programas para asegurar la formación en disciplinas o roles diferentes al de estudio.

\section{Discusión}

Según los resultados de los expertos, los alumnos se vinculan a entornos de atención clínica, sin haber interiorizado los roles, compromisos y responsabilidades de cada miembro del equipo interprofesional. Es decir, se reúnen para tomar decisiones y construir soluciones bajo la perspectiva de su propia identidad profesional. Para una mayor integración de la atención, es deseable aprender conceptos, lenguaje, valores y regulaciones de cada miembro del equipo interprofesional atendiendo lo que la propia definición del concepto establece: se conocen y se relacionan entre sí (WHO, 2010). Según los marcos de referencia presentados, es deseable reconocer las responsabilidades propias del rol y diferenciarlas de aquéllas que forman parte de la colaboración, antes de incorporarse a entornos profesionales reales en los cuales el paciente forma parte del proceso de atención.

Cuando se diseñó el instrumento se planteó con carácter incremental que de acuerdo a la propuesta de Harden (2007) y Olivares et al. (2016). Esta idea implica que se logra un escalón superior cuando se alcanza uno previo. Sin embargo, gracias a las aportaciones de los expertos, se establece que la taxonomía 1) Identifican, 2) Colaboran, 3) Toman decisiones y 4) Construyen soluciones es una implementación dinámica en la cual se construyen decisiones para la atención del paciente, aunque no se conozca el rol del otro miembro del equipo interprofesional. El presente estudio es un piloto que tiene la limitación de haber abordado un equipo acotado de expertos, por lo cual se considera la necesidad de avanzar en la aplicación masiva del instrumento y de esta forma continuar con la reflexión sobre la necesidad de incidir de forma más rigurosa en los contenidos de cada uno de los programas sobre los roles de los miembros del equipo de salud.

\section{Conclusión}

La propuesta presentada integra conceptos de identidad profesional, competencias y de calidad educativa para la implementación de la educación interprofesional en programas de salud. El instrumento final (Anexo 1) cuenta con contenidos comprensibles y adecuados para cumplir con sus propósitos de autodiagnóstico acerca de la formalización de la implementación de competencias interprofesionales. En este sentido el instrumento facilita la reflexión sobre la incidencia de los programas de salud de la institución hacia definición de las competencias, los mecanismos de comunicación a la comunidad académica y las acciones para asegurar la formación de los alumnos en entornos de alta autenticidad tomando en cuenta un enfoque interprofesional.
La educación interprofesional implica mucho más de reunir a varias profesiones a solucionar problemas en temas de salud. Una verdadera integración involucra múltiples actividades de inserción en el plan de estudios que le permiten su definición, implementación, despliegue y mejora continua o innovación. Existen múltiples barreras para limitar los contenidos a estructuras primordialmente disciplinares, sin embargo, es evidente que la colaboración y el trabajo en equipo permite una dinámica de trabajo armoniosa, efectiva y eficaz para los propósitos propios y de los demás.

\section{Reconocimientos}

No se recibieron fondos para el desarrollo del presente trabajo. SLOO realizó introducción, propuesta de las competencias, escala, entrevistas. Mientras que CSLC efectuó método, entrevistas y adecuación del instrumento.

\section{Referencias}

Amelung V, Stein V, Suter E, Goodwin N, Nolte E. \& Balicer R. (2021). Handbook integrated care (2nd edition ed.). Springer.

Bloom, BS, Krathwohl, DK. \& Masia BB. (1972) Taxonomy of educational objectives: The classification of educational goals. Longman, London.

Bogossian F. \& Craven D. (2020).. A review of the requirements for interprofessional education and interprofessional collaboration in accreditation and practice standards for health professionals in Australia. Journal of Interprofessional Care 35, 691 -700.

Cruess SL, Cruess RL. \& Steinert Y. (2019). Supporting the development of a professional identity: General principles. Medical Teacher 41, 641-649.

Díaz-Barriga AF. \& Hernández Rojas G. (2002). Estrategias docentes para un aprendizaje significativo: Una interpretación constructivista. McGraw-Hill, México.

Downing S. (2003). Validity: on meaningful interpretation of assessment data. Medical Education 37, 830-837.

Fitzgerald A. (2020). Professional identity: A concept analysis. Nursing Forum 55, 447-472.

Freeland J. \& Charania M. (2021). 5 steps for building \& strengthening students' networks. Chistensen Institute. Accedido en: https://www. christenseninstitute.org/publications/5-steps-for-building-and-strengthening-students-networks/ el 03 de agosto de 2021.

Gordon F. \& Walsh C. (2005). A Framework for Interprofessional Capability Developing Students of Health and Social Care as CoIlaborative Workers. Journal of Integrated Care 13, 26-33. 
Harden RM. (2007). Outcome-based education - the ostrich, the peacock and the beaver. Medical Teacher 29, 666-671.

Haynes SN, Richard D. \& Kubany E. (1995). Content validity in psychological assessment methods; A functional approach to concepts and methods. Pyschological Assessment 7, 238-247.

Hernández-Sampieri R, Fernández-Collado C y Baptista-Lucio P. (2018). Metodología de la investigación: Las rutas cuantitativa, cualitativa y mixta. McGrawHill, México.

Illingworth P. \& Chelvanayagam S. (2017). The benefits of interprofessional education 10 years on. British Journal of Nursing 26, 813-818.

IPEC. (2016). Interprofessional Education Collaborative: Connecting health professions for better care. Accedido en: https://www.ipecollaborative.org/ 15 de julio de 2021.

Juárez-Hernández LG. \& Tobón S. (2018). Análisis de los elementos implícitos en la validación de contenido de un instrumento de investigación. Espacios 39, 23-30.

Martínez-Corona J, Palacios-Almón G. \& Juárez-Hernández L. (2020). Diseño y validación del instrumento: Enfoque directivo en la gestión para resultados en la sociedad del conocimiento. Revista Espacios 41, 13-26.

Marzano RJ. (2001). Designing a new taxonomy of educational objectives. SAGE Publications.

Ohland MW, Loughry ML, Woehr D, Bullard LG, Felder R, Finelli CJ, Layton RA. Pomeranz HR. \& Schucker DG. (2012). The Comprehensive Assessment of Team Member Effectiveness: Development of a Behaviorally Anchored Rating Scale for Self- and Peer Evaluation. Academy of Management Learning \& Education 11, 609-630.

Olivares Olivares SL. (2021). Aprendizaje Basado en Retos: Transformando la educación en salud. McGraw Hill, México. Accedido en: https://www.mheducation.com.mx/vs-aprendizaje-basado-en-retos-transformar-la-educacion-en-salud-9786071515940-latam el 20 de julio de 2021.

Olivares Olivares SL, López Islas JR, Pineda Garín MJ, Rodríguez Chapa J, Aguayo Hernández C \& Peña Ortega L. (2021). Modelo Educativo Tec21: Retos para una vivencia que transforma. Editorial Digital Tecnológico de Monterrey, México. Accedido en: https:// www.amazon.com.mx/dp/B09C6JN1TY/ 03 de agosto de 2021.
Olivares SL, Rivera N, López MV \& Turrubiates ML. (2020). Etapas de la identidad para ser profesionista: evolución de las expectativas de los retos académicos a lo largo de la carrera. Formación Universitaria 13, 11-20.

Olivares-Olivares SL, Garza-Cruz A y Valdez-García JE. (2016). Etapas del modelo incremental de calidad: un análisis de las escuelas de medicina en México. Revista Investigación en Educación Médica 5, 24-31.

Reeves S, Perrier L, Goldman J, Freeth D. \& Zwarenstein M. (2013). Interprofessional education: effects on professional practice and healthcare outcomes (update). Cochrane Database of Systematic Reviews 28, CD002213.

Rodríguez Medina MA, Poblano-Ojinaga ER, Alvarado Tarango L, González Torres A. \& Rodríguez Borbón MI. (2021). Validación por juicio de expertos de un instrumento de evaluación para evidencias de aprendizaje conceptual. Revista Iberoamericana para la Investigación y Desarrollo Educativo 12, 13-19.

Thistlethwaite JE, Kumar K \& Roberts C. (2016). Becoming interprofessional: Professional identity formation in the health professions. In Teaching medical professionalism: Supporting the development of a professional identity, ed. Crues RT Cruess SR \& Steinert Y, pp 140154. Cambridge Universtiy Press, United Kingdom.

Vermunt J, Ilie S \& Vignoles A. (2018). Building the foundations for measuring learning gain in higher education: a conceptual framework and measurement instrument. Higher Education Pedagogies 3, 266-301.

WHO (2010). Framework for action on Interprofessional Education \& collaborative practice. World Health Organization. Health Professions Networks Nursing \& Midwifery Human Resources for Health. Accedido en: https://www.who.int/publications/i/item/ framework-for-action-on-interprofessional-education-collaborative-practice el 15 de agosto de 2021.

Zorek J. \& RaehI C. (2013). Interprofessional education accreditation standards in the USA: A comparative analysis. Journal of Interprofessional Care 27, 123-130. 
Anexo 1: Autodiagnóstico de Integración de la Educación Interprofesional en los programas de salud.

\begin{tabular}{|c|c|c|c|c|c|c|}
\hline $\begin{array}{l}\text { El perfil de egreso contie- } \\
\text { ne competencias asocia- } \\
\text { das con la taxonomía de } \\
\text { competencias interpro- } \\
\text { fesionales }\end{array}$ & Competencias desagregadas & $\begin{array}{l}\text { Están definidas } \\
\text { y mapeadas en } \\
\text { el programa. } \\
\text { Forman } \\
\text { parte de las } \\
\text { intenciones y } \\
\text { contenidos de } \\
\text { los cursos. }\end{array}$ & $\begin{array}{c}\text { Existen } \\
\text { mecanismos } \\
\text { de comunica- } \\
\text { ción efectivos } \\
\text { y eficientes } \\
\text { hacia toda la } \\
\text { comunidad } \\
\text { académica } \\
\end{array}$ & $\begin{array}{c}\text { Se asegura } \\
\text { la } \\
\text { formación / } \\
\text { educación } \\
\text { en los alum- } \\
\text { nos }\end{array}$ & $\begin{array}{l}\text { Se evalúan } \\
\text { sistemáticamente. } \\
\text { Hay indicadores } \\
\text { de progreso por } \\
\text { alumno y } \\
\text { programa. }\end{array}$ & $\begin{array}{c}\text { Se establecen } \\
\text { estrategias de } \\
\text { mejora e } \\
\text { innovación a } \\
\text { intervalos } \\
\text { adecuados }\end{array}$ \\
\hline \multirow{4}{*}{$\begin{array}{l}\text { Identifican los roles, com- } \\
\text { promisos y responsabilida- } \\
\text { des de cada miembro del } \\
\text { equipo interprofesional }\end{array}$} & $\begin{array}{l}\text { Conocen lo que hacen los otros } \\
\text { profesionales de la salud }\end{array}$ & 1 & 2 & 3 & 4 & 5 \\
\hline & $\begin{array}{l}\text { Distinguen las funciones propias } \\
\text { de las de otros profesionales }\end{array}$ & 1 & 2 & 3 & 4 & 5 \\
\hline & $\begin{array}{l}\text { Tienen claridad sobre sus valores } \\
\text { y preferencias y actúa conforme } \\
\text { a los mismos }\end{array}$ & 1 & 2 & 3 & 4 & 5 \\
\hline & $\begin{array}{l}\text { Están conscientes de las regu- } \\
\text { laciones de otras profesiones } \\
\text { de los miembros del equipo } \\
\text { interprofesional para lograr una } \\
\text { atención informada }\end{array}$ & 1 & 2 & 3 & 4 & 5 \\
\hline \multirow{3}{*}{$\begin{array}{l}\text { Colaboran con profesio- } \\
\text { nalismo con cada uno de } \\
\text { los miembros del equipo } \\
\text { inteprofesional }\end{array}$} & $\begin{array}{l}\text { Construyen o amplian sus redes } \\
\text { académicas, sociales y personales } \\
\text { afines a sus intereses, tanto de } \\
\text { carácter formal como informal }\end{array}$ & 1 & 2 & 3 & 4 & 5 \\
\hline & $\begin{array}{l}\text { Desarrollan códigos de lenguaje, } \\
\text { valores y actitudes en relación a } \\
\text { un grupo interprofesional }\end{array}$ & 1 & 2 & 3 & 4 & 5 \\
\hline & $\begin{array}{l}\text { Respetan la aportación de cada } \\
\text { uno de los miembros del equipo de } \\
\text { atención (incluyendo el paciente) } \\
\text { en la toma de decisiones, consi- } \\
\text { derando la ética de la práctica } \\
\text { interprofesional }\end{array}$ & 1 & 2 & 3 & 4 & 5 \\
\hline \multirow{2}{*}{$\begin{array}{l}\text { Toman decisiones de forma } \\
\text { conjunta para la atención } \\
\text { centrada en el paciente en } \\
\text { el equipo interprofesional }\end{array}$} & $\begin{array}{l}\text { Promueven una atención coor- } \\
\text { dinada en donde los servicios } \\
\text { comparten información clínica } \\
\text { y administran la transición de } \\
\text { pacientes entre las diferentes } \\
\text { unidades o grupos. }\end{array}$ & 1 & 2 & 3 & 4 & 5 \\
\hline & $\begin{array}{l}\text { Se centran en la valoración de las } \\
\text { necesidades del paciente a partir } \\
\text { de colaboración, participación y } \\
\text { comunicación en equipos inter- } \\
\text { profesionales }\end{array}$ & 1 & 2 & 3 & 4 & 5 \\
\hline \multirow{3}{*}{$\begin{array}{l}\text { Construyen soluciones de } \\
\text { innovación y mejora en } \\
\text { equipo interprofesional }\end{array}$} & $\begin{array}{l}\text { Generan transformaciones asocia- } \\
\text { das con la profesión de su elección } \\
\text { dentro de un contexto académico } \\
\text { u organizacional. }\end{array}$ & 1 & 2 & 3 & 4 & 5 \\
\hline & \begin{tabular}{|l|} 
Proveen servicios orientados \\
a grupos específicos con una \\
atención longitudinal
\end{tabular} & 1 & 2 & 3 & 4 & 5 \\
\hline & $\begin{array}{l}\text { Los servicios que se proveen } \\
\text { consideran a cada uno de los } \\
\text { miembros, incluyendo el paciente } \\
\text { y sus familias, para la toma de } \\
\text { decisiones alrededor del proceso } \\
\text { de intervención. }\end{array}$ & 1 & 2 & 3 & 4 & 5 \\
\hline
\end{tabular}

